Bull. Austral. Math. Soc.

49L $25,35 \mathrm{~B} 37$

VOL. $53(1996) \quad[51-62]$

\title{
ON THE BELLMAN EQUATIONS WITH VARYING CONTROL
}

\author{
SHIGEAKI KOIKE
}

\begin{abstract}
The value function is presented by minimisation of a cost functional over admissible controls. The associated first order Bellman equations with varying control are treated. It turns out that the value function is a viscosity solution of the Bellman equation and the comparison principle holds, which is an essential tool in obtaining the uniqueness of the viscosity solutions.
\end{abstract}

\section{INTRODUCTION}

In this article, we consider the following Bellman equations of first order:

$$
\sup _{a \in A(x)}\{\lambda u(x)-\langle g(x, a), D u(x)\rangle-f(x, a)\}=0 \quad \text { for } x \in \Omega
$$

where $\lambda>0$ is a positive constant, $A(x)$ is a subset of $A$ for each $x \in \Omega$, and $\boldsymbol{g}: \bar{\Omega} \times A \rightarrow \mathbf{R}^{n}$ and $f: \bar{\Omega} \times A \rightarrow \mathbf{R}$ are given continuous functions. Here, $\Omega \subset \mathbf{R}^{n}$ is a bounded open set and $A$ a compact set in $\mathbf{R}^{N}$ (for $n, N \in \mathbf{N}$ ).

In the case when $A(\cdot)$ does not depend on $x \in \Omega$, many authors have investigated the Bellman equation (1.1) particularly via the viscosity solution approach. We only refer to Fleming and Soner [3] and the references therein.

In applications, for example in [1] and references therein, it is important to study the case when $A$ may depend on the position $x$. In fact, it is well-known that, if we consider minimisation of a cost functional at each point $x \in \Omega$ over all controls in $A$, then the value function satisfies (1.1) with $A(x) \equiv A$ (in the viscosity sense). On the other hand, if the available controls depend on the position of the associated states, then it will turn out that the associated value function satisfies (1.1) with varying $A(x)$.

\footnotetext{
Received 16th March, 1995

Supported in part by Grant-in-Aid for Scientific Research (No. 06740094), Ministry of Education, Science and Culture and by Visiting Fellowship in the Centre for Mathematics and its Applications of the Australian National University. This work was done while the author was visiting at the Centre for Mathematics and its Applications in the Australian National University at Canberra. He expresses his sincere thanks to Professor Neil S. Trudinger and the CMA for their warm hospitality. He would like to thank Dr. Matthew R. James in the Department of Systems Engineering of the Australian National University for leading him to an interest in this research.
}

Copyright Clearance Centre, Inc. Serial-fee code: 0004-9729/96 \$A2.00+0.00. 
Here we shall mainly treat the problems when $A(\cdot)$ moves "smoothly". In fact, as we shall see in an example, we can not expect in general that the associated value function is continuous if we do not make the smoothness assumption.

Our first aim here is to present a general setting to treat the Bellman equations with varying control. In our framework, we show that the associated value function is a viscosity solution of (1.1). We also establish the comparison principle for viscosity solutions of (1.1), which implies the uniqueness and continuity of viscosity solutions. In order to make our statement and argument simpler, we shall only consider the Dirichlet boundary value problem although other boundary conditions may be treated. We notice that, in order to apply the comparison principle to the uniqueness result under the Dirichlet condition, we need to know some continuity of viscosity solutions near the boundary. Since it seems another task, we shall not give the uniqueness result in this note. We refer to $[4,2]$ and references therein for the details.

We shall moreover discuss the case when $A(\cdot)$ may vary rather drastically but the comparison principle holds. It will give new scope to the formulation of the state constraint problem introduced in [6].

This paper is organised as follows: Section 2 is devoted to defining our value function and to giving the dynamic programming principle for it. In Section 3 we show that, under appropriate hypotheses, the value function is a viscosity solution of the Bellman equation (1.1). In Section 4, we present our comparison principle for viscosity solutions under the Dirichlet condition, which implies that the value function is the unique viscosity solution of (1.1). In the final section, we discuss a special case when $A(\cdot)$ does not vary continuously.

\section{VAlue function}

First of all, we make at least the following regularity assumption for the given functions throughout this paper:

$$
\begin{cases}\text { (1) } & f \in C(\bar{\Omega} \times A ; \mathbf{R}), g \in C\left(\bar{\Omega} \times A ; \mathbf{R}^{n}\right), h \in C(\partial \Omega ; \mathbf{R}) . \\ & \text { There is } \omega \in C\left(\mathbf{R}^{+} ; \mathbf{R}^{+}\right) \text {with } \omega(0)=0 \text { such that } \\ \text { (2) } & |f(x, a)-f(\widehat{x}, \widehat{a})| \leqslant \omega(|x-\widehat{x}|+|a-\widehat{a}|) \\ \text { (3) } & |g(x, a)-g(x, \widehat{a})| \leqslant \omega(|a-\widehat{a}|) \\ & \text { for } x, \widehat{x} \in \bar{\Omega} \text { and } a, \widehat{a} \in A . \\ \text { (4) } \sup _{a \in A}\left\{\|g(\cdot, a)\|_{W^{1, \infty}(\Omega)}+\|f(\cdot, a)\|_{L^{\infty}(\Omega)}\right\}<\infty .\end{cases}
$$

Here, $h$ will be the Dirichlet data on $\partial \Omega$.

We shall present the value function, which satisfies (1.1) in the viscosity sense under appropriate hypotheses. We shall only consider the Dirichlet type boundary condition 
for simplicity although other boundary conditions (oblique, state-space constraint et cetera) may be treated under suitable hypotheses.

We denote by $\mathcal{A}$ the set of all measurable functions $\alpha:[0, \infty) \rightarrow A$. For each $\alpha \in \mathcal{A}$ and $x \in \bar{\Omega}$, we denote by $X(\cdot ; x, \alpha)$ the unique solution of

$$
\left\{\begin{array}{l}
X^{\prime}(t)=g(X(t), \alpha(t)) \quad \text { for } t>0 \\
X(0)=x
\end{array}\right.
$$

We occasionally use $a \in A$ as an element of $\mathcal{A}$. For instance, we denote by $X(\cdot ; x, a)$ for $a \in A$ the solution of $(2.1)$ with $\alpha(\cdot) \equiv a$.

We denote by $\tau(x, \alpha) \geqslant 0$ the first hitting time on $\partial \Omega$ of $X(\cdot ; x, \alpha)$;

$$
\tau(x, \alpha)=\inf \{t \geqslant 0 \mid X(t ; x, \alpha) \in \partial \Omega\} .
$$

Now we define the admissible control set, over which we shall minimise a cost functional:

$$
\mathcal{A}(x)=\{\alpha \in \mathcal{A} \mid \alpha(t) \in A(X(t ; x, \alpha)) \text { for almost all } t \in[0, \tau(x, \alpha))\} .
$$

The restriction in the definition of $\mathcal{A}(x)$ causes the $x$-dependence of the control set in the Bellman equation (1.1) (that is $A(x)$ ), which the associated value function satisfies. We notice that this restriction is often required in practical control problems. We refer to [1] for our motivation. In what follows, we suppose the following:

$$
\mathcal{A}(x) \neq \emptyset \quad \text { for all } \quad x \in \Omega \text {. }
$$

We also denote the admissible control set up to a time $s>0$ by the following:

$$
\mathcal{A}^{*}(x)=\{\alpha \in \mathcal{A} \mid \alpha(t) \in A(X(t ; x, \alpha)) \text { for almost all } t \in[0, s)\} \text {. }
$$

Note that (A2) implies that, for each $x \in \Omega$, there exists $s>0$ such that $\mathcal{A}^{\bullet}(x) \neq \emptyset$.

Our value function is as follows:

$$
V(x)=\inf _{\alpha \in \mathcal{A}(x)}\left\{\int_{0}^{\tau(x, \alpha)} e^{-\lambda t} f(X(t ; x, \alpha), \alpha(t)) d t+e^{-\lambda \tau(x, \alpha)} h(X(\tau(x, \alpha) ; x, \alpha))\right\} .
$$

It is easy to find a bound for the value function:

Proposition 2.1. Under assumptions (A1) and (A2), we have

$$
\|V\|_{L^{\infty}(\Omega)}<\infty \text {. }
$$

By employing the standard argument, we easily obtain the dynamic programming principle, which will be useful in deriving our verification theorem.

Proposition 2.2. (See [6].) Under assumptions (A1) and (A2), for each $x \in \Omega$, there is a small $s_{0}>0$ such that

$$
V(x)=\inf _{\alpha \in \mathcal{A}^{\prime}(x)}\left\{\int_{0}^{s} e^{-\lambda t} f(X(t ; x, \alpha), \alpha(t)) d t+e^{-\lambda \varepsilon} V(X(s ; x, \alpha))\right\}
$$

for $s \in\left(0, s_{0}\right)$. 


\section{VERIFICATION}

In this section, we verify that the value function in the previous section is indeed a viscosity solution of (1.1) under appropriate hypotheses.

We denote by $A(x)$ for $x \in \Omega$ the following subset of $A$ :

$$
A(x)=\left\{\begin{array}{l|l}
a \in A & \begin{array}{c}
\text { There is } r>0 \text { such that } a \in A(X(t ; y, a)) \\
\text { for } t \in[0, r) \text { and } y \in B_{r}(x) \subset \Omega .
\end{array}
\end{array}\right\}
$$

We denote by $B_{s}(z)$ the standard open ball of $\mathbf{R}^{n}$ or $\mathbf{R}^{N}$ with the radius $s>0$ and the centre $z \in \mathbf{R}^{n}$ or $z \in \mathbf{R}^{N}$.

We shall assume the following hypothesis, which implies (A2):

$$
A(x) \neq \emptyset \text { for all } x \in \Omega
$$

We shall utilise the following notation for simplicity:

$$
H_{a}(x, r, p)=\lambda r-\langle g(x, a), p\rangle-f(x, a) \text { and } H(x, r, p)=\sup _{a \in A(x)} H_{a}(x, r, p)
$$

for $a \in A$ and $(x, r, p) \in \Omega \times \mathbf{R} \times \mathbf{R}^{n}$.

We now give our definition of viscosity solution for (1.1).

Definition: We call $u: \Omega \rightarrow \mathbf{R}$ a viscosity subsolution (respectively, supersolution) of (1.1) if, for any $\phi \in C^{1}(\Omega)$ and for any $x \in \Omega$ such that $u^{*}-\phi$ (respectively, $u_{*}-\phi$ ) attains its local maximum (respectively, minimum) at $x$,

$$
\left.H_{*}\left(x, u^{*}(x), D \phi(x)\right) \leqslant 0 \quad \text { (respectively, } \quad H^{*}\left(x, u_{*}(x), D \phi(x)\right) \geqslant 0\right) .
$$

We also call $u: \Omega \rightarrow \mathbf{R}$ a viscosity solution of (1.1) if it is both a viscosity suband supersolution of (1.1).

We remark that, for real valued functions, we denote their upper and lower semicontinuous envelopes, respectively, by putting the superscript $*$ and the subscript $*$ as in the standard notation of the theory of viscosity solutions. We refer to [2] for these.

In applications, it is important to treat the following case (see [1]):

Example 1. Set $A=[-1,1] \subset \mathbf{R}, k_{i} \in C(\Omega)$ (for $i=1,2$ ) satisfying $-1 \leqslant k_{1}(x)<$ $k_{2}(x) \leqslant 1$ for $x \in \bar{\Omega}, A(x)=\left\{a \in A \mid k_{1}(x)<a<k_{2}(x)\right\}$, and $g(x, a) \equiv a g_{1}(x)+g_{2}(x)$ for some $g_{i} \in W^{1, \infty}(\Omega)$ (for $i=1,2$ ).

It is easy to check that this example satisfies (A1) and (A2').

We also give an example which satisfies (A1) and (A2') but the value function is discontinuous. The example indicates that we can not obtain the comparison principle in general since it implies the continuity of the value function. 
EXAmple 2. Consider $\Omega=(-1,1), \lambda=1, h \equiv 0$ and $A=\{1,2\}$. Set $g(x, 1)=$ $1, g(x, 2)=-1, f(x, 1)=1$ and $f(x, 2)=b \in(0,1)$ for $x \in(-1,1)$. We define

$$
A(x)= \begin{cases}\{1\} & \text { for } x \in[0,1) \\ \{1,2\} & \text { for } x \in(-1,0)\end{cases}
$$

Then, it is easy to check that the value function of (1.1) of the above case is explicitly given by

$$
V(x)= \begin{cases}1-e^{x-1} & \text { for } x \in[0,1] \\ b\left(1-e^{-x-1}\right) & \text { for } x \in[-1,0)\end{cases}
$$

Thus, $V$ is discontinuous at $x=0$. It is also easy to verify that this is a (discontinuous) viscosity solution of (1.1).

In order to verify that the value function is a viscosity solution of (1.1), we assume the following condition on $A(x)$ :

$$
\left\{\begin{array}{l}
\text { For } z \in \Omega, \varepsilon>0, \text { there are } r, s>0 \text { satisfying the following: } \\
\text { For any } x \in B_{r}(z) \text { and } a \in A(x) \text {, there is } \widehat{a} \in B_{\varepsilon}(a) \cap A \\
\text { such that } \widehat{a} \in \mathcal{A}(X(t ; x, \widehat{a})) \text { for } t \in[0, s)
\end{array}\right.
$$

This condition indicates that we may not choose $s>0$ so that $a \in \mathcal{A}^{s}(x)$ uniformly in $a \in A(x)$ but we can approximate any $a \in A(x)$ by $\hat{a} \in \mathcal{A}^{*}(x)$ with a small $s>0$ independent of $a \in A(x)$. We notice that assumption (A3) holds for Examples 1 and 2.

THEOREM 3.1. Under assumptions (A1), (A2') and (A3), the value function $V$ is a viscosity solution of (1.1).

Proof: We only give the subsolution assertion since the other assertion can be proved similarly.

If $V$ is not a viscosity subsolution of (1.1), then we may suppose that there are $x \in \Omega$ and $\phi \in C^{1}$ such that $V^{*}(x)-\phi(x)=\max _{y \in \bar{\Omega}} V^{*}(y)-\phi(y)=0$ and that

$$
H_{*}(x, \phi(x), D \phi(x)) \geqslant \gamma \quad \text { for some constant } \quad \gamma>0 \text {. }
$$

For small $\delta>0$, we can choose $x_{\delta} \in B_{\delta}(x)$ such that

$$
0 \geqslant V\left(x_{\delta}\right)-\phi\left(x_{\delta}\right) \geqslant-\delta
$$

Moreover, by (A1)-(2) and (4), we have

$$
H\left(x_{\delta}, \phi\left(x_{\delta}\right), D \phi\left(x_{\delta}\right)\right) \geqslant \frac{\gamma}{2} .
$$


Hence, we can find $a_{\delta} \in A\left(x_{\delta}\right)$ such that

$$
H_{a_{\delta}}\left(x_{\delta}, \phi\left(x_{\delta}\right), D \phi\left(x_{\delta}\right)\right) \geqslant \frac{\gamma}{4}
$$

By using the uniform continuity with respect to $a \in A$ of the given functions in (A1) $-(2)$ and (3), (A3) implies that there are $\widehat{r}>0$ and $\widehat{a}_{\delta} \in \mathcal{A}^{r}\left(x_{\delta}\right)$ such that

$$
H_{\widehat{a}_{\delta}}\left(x_{\delta}, \phi\left(x_{\delta}\right), D \phi\left(x_{\delta}\right)\right) \geqslant \frac{\gamma}{8}
$$

By (A1)-(2) and (4), we find $r \in(0, \widehat{r}]$ such that

$$
H_{\widehat{a}_{\delta}}\left(X_{\delta}(t), \phi\left(X_{\delta}(t)\right), D \phi\left(X_{\delta}(t)\right)\right) \geqslant \frac{\gamma}{16} \text { for } t \in(0, r)
$$

where $X_{\delta}(t)=X\left(t ; x_{\delta}, \widehat{a}_{\delta}\right)$. Multiplying (3.2) by $e^{-\lambda t}$ and then integrating over $(0, r)$, by $(2.1)$, we arrive at the following:

$$
-e^{-\lambda r} \phi\left(X_{\delta}(r)\right)+\phi\left(x_{\delta}\right)-\int_{0}^{r} e^{-\lambda t} f\left(X_{\delta}(t), \widehat{a}_{\delta}\right) d t \geqslant \frac{\gamma}{16 \lambda}\left(1-e^{-\lambda r}\right)
$$

Hence, by (3.1) and the fact that $V \leqslant \phi$ in $\bar{\Omega}$, we have

$$
-e^{-\lambda r} V\left(X_{\delta}(r)\right)+\delta+V\left(x_{\delta}\right)-\int_{0}^{r} e^{-\lambda t} f\left(X_{\delta}(t), \widehat{a}_{\delta}\right) d t \geqslant \frac{\gamma}{16 \lambda}\left(1-e^{-\lambda r}\right) .
$$

By taking the supremum of the above over all $\alpha \in \mathcal{A}^{r}\left(x_{\delta}\right)$, Propostion 2.2 yields

$$
\delta \geqslant \frac{\gamma}{16 \lambda}\left(1-e^{-\lambda r}\right)
$$

Therefore, for small $\delta>0$, we get a contradiction.

\section{COMPARISON PRINCIPLE}

In this section, we give the comparison principle for viscosity solutions of (1.1), which implies that, if the value function is continuous on the boundary (that is, $V^{*}=V_{*}=h$ on $\partial \Omega$ ), then it is the unique viscosity solution of (1.1) among possibly discontinuous solutions. (In fact, it turns out that such a value function is continuous in $\bar{\Omega}$.) We only refer to [4] for a sufficient condition which implies the continuity of the value function on $\partial \Omega$. 
For our comparison principle, we assume the following hypotheses, stronger than (A1), on the regularity for the given functions:

$\left(\mathrm{A} 1^{\prime}\right)$

$$
\begin{cases}\text { (1) } & \text { There is } K>0 \text { such that } \\ & |g(x, a)-g(\widehat{x}, \widehat{a})| \leqslant K(|x-\widehat{x}|+|a-\widehat{a}|) \\ & \text { for }(x, a),(\widehat{x}, \widehat{a}) \in \bar{\Omega} \times A . \\ \text { (2) } \quad \text { There is } \omega \in C\left(\mathbf{R}^{+} ; \mathbf{R}^{+}\right) \text {with } \omega(0)=0 \text { such that } \\ \\ |f(x, a)-f(x, a)| \leqslant \omega(|x-\widehat{x}|+|a-\widehat{a}|) \\ & \text { for }(x, a),(\widehat{x}, \widehat{a}) \in \bar{\Omega} \times A . \\ \text { (3) } \sup _{a \in A}\left\{\|f(\cdot, a)\|_{L^{\infty}(\Omega)}+\|g(\cdot, a)\|_{L^{\infty}(\Omega)}\right\}<\infty .\end{cases}
$$

We also make the following "locally Lipschitz continuity" assumption on the setvalued mapping $A(\cdot)$ :

$$
\left\{\begin{array}{l}
\text { For any } z \in \Omega \text { and } \varepsilon>0 \text { with } B_{\varepsilon}(z) \subset \Omega, \text { there is } r>0 \\
\text { such that } A(x) \subset A_{r|x-y|}(y) \text { provided } x, y \in B_{\varepsilon}(z),
\end{array}\right.
$$

where $A_{s}(y)=\{a \in A \mid \operatorname{dist}(a, A(y)) \leqslant s\}$ for $s>0$.

Theorem 4.1. Assume ( $\left.A 1^{\prime}\right),\left(A 2^{\prime}\right)$ and (A4) hold. Let $u$ and $v$ be bounded viscosity sub- and supersolutions of (1.1), respectively. If $u^{*} \leqslant v_{*}$ on $\partial \Omega$, then $u^{*} \leqslant v_{*}$ in $\bar{\Omega}$.

ProOF: We shall write $u$ and $v$ instead of $u^{*}$ and $v_{*}$, respectively.

The proof is by contradiction: Suppose $\max _{\bar{\Omega}}(u-v)=\gamma$ for some $\gamma>0$. By a perturbation technique (if necessary), we may find the unique maximum point $z \in \Omega$;

$$
u(z)-v(z)>u(x)-v(x) \quad \text { for } \quad x \in \bar{\Omega} \backslash\{z\} .
$$

Consider $\Phi(x, y)=u(x)-v(y)-\left(|x-y|^{2}\right) / 2 \varepsilon$ for $\varepsilon>0$. Let $\left(x_{\varepsilon}, y_{\varepsilon}\right) \in \bar{\Omega} \times \bar{\Omega}$ be the maximum point of $\Phi$ over $\bar{\Omega} \times \bar{\Omega}$. By the standard argument, we see

$$
\lim _{e \rightarrow 0} x_{e}=\lim _{\varepsilon \rightarrow 0} y_{e}=z \text {, and } \lim _{e \rightarrow 0} \frac{\left|x_{e}-y_{e}\right|^{2}}{\varepsilon}=0 \text {. }
$$

For simplicity, we shall write $x$ and $y$ in place of $x_{\varepsilon}$ and $y_{\varepsilon}$, respectively.

From the definition, for $\delta>0$, we can choose $\left(x_{\delta}, r_{\delta}, p_{\delta}\right),\left(y_{\delta}, s_{\delta}, q_{\delta}\right) \in \Omega \times \mathbf{R} \times \mathbf{R}^{n}$ such that

$$
\begin{gathered}
\lim _{\delta \rightarrow 0} x_{\delta}=x, \lim _{\delta \rightarrow 0} y_{\delta}=y, \lim _{\delta \rightarrow 0}\left(\left|r_{\delta}\right|+\left|s_{\delta}\right|+\left|p_{\delta}\right|+\left|q_{\delta}\right|\right)=0, \\
\delta \geqslant H\left(x_{\delta}, u(x)+r_{\delta},(x-y) / \varepsilon+p_{\delta}\right),
\end{gathered}
$$

and

$$
-\delta \leqslant H\left(y_{\delta}, v(y)+s_{\delta},(x-y) / \varepsilon+q_{\delta}\right) .
$$


From (4.4), we can choose $a_{\delta} \in A\left(y_{\delta}\right)$ such that

$$
-2 \delta \leqslant H_{a_{\delta}}\left(y_{\delta}, v(y)+s_{\delta},(x-y) / \varepsilon+q_{\delta}\right)
$$

By (A4), we choose $r_{0}>0$ such that $a_{\delta} \in A_{r_{0}\left|z_{\delta}-y_{\delta}\right|}\left(x_{\delta}\right)$. Hence, from (4.3) and $\left(\mathrm{A1} 1^{\prime}\right)-(1)$ and $(2)$, we have

$$
\begin{gathered}
\delta+\omega\left(r_{0}\left|x_{\delta}-y_{\delta}\right|\right)+K r_{0}\left|x_{\delta}-y_{\delta}\right|\left(\frac{|x-y|}{\varepsilon}+\left|p_{\delta}\right|\right) \\
\geqslant H_{a_{\delta}}\left(x_{\delta}, u(x)+r_{\delta},(x-y) / \varepsilon+p_{\delta}\right)
\end{gathered}
$$

Combining (4.5) with this, we have

$$
\begin{aligned}
\lambda(u(x)-v(y))-3 \delta & -C\left(\left|r_{\delta}\right|+\left|s_{\delta}\right|+\left|p_{\delta}\right|+\left|q_{\delta}\right|\right) \\
& \leqslant \omega\left(\left|x_{\delta}-y_{\delta}\right|\right)+\omega\left(r_{0}\left|x_{\delta}-y_{\delta}\right|\right)+K\left(1+r_{0}\right) \frac{|x-y|}{\varepsilon}\left|x_{\delta}-y_{\delta}\right| .
\end{aligned}
$$

Here and later, $C$ denotes various positive constants depending only on known quantities. Sending $\delta \rightarrow 0$ with (4.2) in the above, we have

$$
\lambda(u(x)-v(y)) \leqslant \omega(|x-y|)+\omega\left(r_{0}|x-y|\right)+K\left(1+r_{0}\right) \frac{|x-y|^{2}}{\varepsilon} .
$$

Using $\Phi(x, y) \geqslant \gamma$ and sending $\varepsilon \rightarrow 0$ in the above with (4.1), we have $\lambda \gamma \leqslant 0$, which is a contradiction.

\section{A CASE Without assumption (A4)}

In this section, we discuss the case when $A(\cdot)$ does not satisfy the continuity assumption (A4). As indicated in Example 2, we can not expect in general to obtain the comparison principle.

For simplicity, in this section, we only consider the case when $\Omega$ consists of several nice components: Set $L \equiv\{1,2, \ldots, l\}$ for some integer $l \in \mathbf{N}$.

$$
\left\{\begin{array}{l}
\text { (1) There are }\left\{\Omega_{k}\right\}_{k \in L} \text { such that } \Omega_{k} \cap \Omega_{\widehat{k}}=\emptyset \text {, for } k \neq \hat{k}, \\
\operatorname{int}\left(\Omega_{k}\right) \neq \emptyset, \text { for } k \in L \text {, and } \bigcup_{k \in L} \Omega_{k}=\Omega . \\
\text { (2) For any } z \in \Omega_{k} \text { and } \varepsilon>0, \text { there is } r>0 \\
\text { such that } A(x) \subset A_{r|x-y|}(y) \text { provided } x, y \in B_{\varepsilon}(z) \cap \Omega_{k} .
\end{array}\right.
$$

We also suppose that at most two of $\left\{\bar{\Omega}_{k}\right\}_{k \in L}$ have nonempty intersection and, at such a point, we assume some order of the sets of available controls in the following 
manner:

$$
\begin{aligned}
& \left\{\begin{array}{l}
\text { (1) } \bigcap_{i=1}^{3} \bar{\Omega}_{k_{i}}=\emptyset \text { for any distinct } k_{1}, k_{2}, k_{3} \in L . \\
\text { (2) For } z \in \partial \Omega_{k_{1}} \cap \partial \Omega_{k_{2}} \text { with } k_{1}<k_{2} \text { and for } \varepsilon>0,
\end{array}\right. \\
& \text { there is } r>0 \text { such that } A(y) \subset A_{\varepsilon}(x) \text { holds } \\
& \text { for } x \in \Omega_{k_{1}} \cap B_{r}(z) \text { and } y \in \Omega_{k_{2}} \cap B_{r}(z) \text {. }
\end{aligned}
$$

We moreover assume certain condition on the vector fields $g$ near the intersection of $\left\{\partial \Omega_{k}\right\}_{k \in L}$ :

$$
\left\{\begin{array}{l}
\text { For } z \in \partial \Omega_{k_{1}} \cap \partial \Omega_{k_{2}} \text { with } k_{1}<k_{2}, \text { there are } r, \theta \in(0,1) \\
\text { and } \xi \in S^{n-1} \text { satisfying the following properties: } \\
\text { (1) } \bigcup_{0<t<r} B_{t \theta}(x+t \xi) \subset \Omega_{k_{1}} \text { for } x \in \Omega_{k_{1}} \cap B_{r}(z) \\
(2) \quad g(x, a) \in \bigcup_{t>0} B_{t \theta}(-t \xi) \text { for any } a \in A(x) \backslash A(y) \\
\text { with } x \in \Omega_{k_{1}} \cap B_{r}(z) \text { and } y \in \Omega_{k_{2}} \cap B_{r}(z) .
\end{array}\right.
$$

Notice that Example 2 does not satisfy (A6). In fact, this condition requires that $A(x)\left(x \in \Omega_{k_{1}} \cap B_{r}(z)\right)$ can only have more controls than $A(y)\left(y \in \Omega_{k_{2}} \cap B_{r}(z)\right)$ which directs inside $\Omega_{k_{2}}$.

We also suppose that the vector fields on the intersection of $\left\{\bar{\Omega}_{k}\right\}_{k \in L}$ do not vanish:

$$
\left\{\begin{array}{l}
\text { There are } \tau>0 \text { and } r>0 \text { such that, if } z \in \partial \Omega_{k_{1}} \cap \partial \Omega_{k_{2}} \\
\text { with } k_{1}<k_{2}, \text { then }|g(z, a)| \geqslant \tau \text { for any } a \in A(x) \backslash A(y) \\
\text { with } x \in \Omega_{k_{1}} \cap B_{r}(z) \text { and } y \in \Omega_{k_{2}} \cap B_{r}(z) .
\end{array}\right.
$$

We remark that, under these assumptions, we can treat the state-space constraint problem in control theory as follows:

EXAMPLE 3. Let $\Omega_{1} \subset \Omega$ be a (smooth) open set with $\bar{\Omega}_{1} \subset \Omega$ and $\Omega_{2} \equiv \Omega \backslash \Omega_{1}$. Suppose $A(x)=A$ for $x \in \Omega_{1}$ and $A(x)=\left\{a \in A \mid\langle g(x, a), n(x)\rangle<0\right.$ for $\left.x \in \partial \Omega_{1}\right\} \neq$ $\emptyset$ for $x \in \Omega_{2}$. Here, $n(x)$ denotes the exterior unit normal vector at $x \in \partial \Omega_{1}$ relative to $\Omega_{1}$. If we restrict ourselves to considering the value function in $\bar{\Omega}_{1}$, then it is the unique viscosity solution of (1.1) in $\bar{\Omega}_{1}$ under suitable hypotheses. We refer to [6] for the details of the state-space constraint problems and their appropriate formulation.

Now we shall present our comparison principle:

Theorem 5.1. Assume $\left(A 1^{\prime}\right),\left(A 2^{\prime}\right),\left(A 4^{\prime}\right)$ and $(A 5)-(A 7)$ hold. Let $u$ and $v$ be bounded viscosity sub- and supersolutions of (1.1), respectively. If $u^{*} \leqslant v_{*}$ on $\partial \Omega$, then $u^{*} \leqslant v_{*}$ in $\bar{\Omega}$.

Proof: We shall write $u$ and $v$ instead of $u^{*}$ and $v_{*}$, respectively, for simplicity. 
The proof is by contradiction: Suppose $\max _{\overline{\mathbf{n}}}(u-v)=\gamma>0$. We may suppose that there is a unique maximum point $z \in \Omega$ as in the proof of Theorem 4.1.

If $z \in \operatorname{int}\left(\Omega_{k}\right)$ for some $k \in L$, then the same argument as in the proof of Theorem 4.1 leads to a contradiction. Thus, we may assume $z \in \partial \Omega_{1} \cap \partial \Omega_{2}$.

Now we choose $r, \theta \in(0,1)$ and $\xi \in S^{n-1}$ satisfying (A6) for $z \in \partial \Omega_{1} \cap \partial \Omega_{2}$. Using this notation, we can find the following function:

Lemma 5.2. (Lemma 6.1 in [7].) There exists $\phi \in C^{1}\left(\mathbf{R}^{n}\right)$ such that

$$
\sigma^{-1}|x|^{2} \leqslant \phi(x) \leqslant \sigma|x|^{2},|D \phi(x)| \leqslant \sigma|x| \quad \text { for some } \quad \sigma>1,
$$

and

$$
\langle\eta, D \phi(x)\rangle \geqslant 0 \text { for } \eta \in \bigcup_{t>0} B_{t \theta}(-t \xi) \text { and } x \notin \bigcup_{t>0} B_{t \theta}(t \xi)
$$

Define the function $\Phi: \bar{\Omega} \times \bar{\Omega} \rightarrow \mathbf{R}$ by

$$
\Phi(x, y)=u(x)-v(y)-\frac{1}{\varepsilon} \phi(x-y)+\mu\langle\xi, x-y\rangle,
$$

where $\mu>0$ will be fixed later.

Let $\left(x_{\varepsilon}, y_{\varepsilon}\right) \in \bar{\Omega} \times \bar{\Omega}$ be the maximum point of $\Phi$ over $\bar{\Omega} \times \bar{\Omega}$. The standard argument implies that

$$
\lim _{\varepsilon \rightarrow 0} x_{e}=\lim _{\varepsilon \rightarrow 0} y_{e}=z, \text { and } \quad \lim _{e \rightarrow 0} \frac{\left|x_{e}-y_{e}\right|^{2}}{\varepsilon}=0 \text {. }
$$

For the sake of simplicity, we shall write $x$ and $y$ instead of $x_{\varepsilon}$ and $y_{e}$.

We first note that the definition of $u$ yields

$$
0 \geqslant H_{*}(x, u(x), D \phi(x-y) / \varepsilon-\mu \xi) \text {. }
$$

Thus, for small $\delta>0$, there exist $\left(x_{\delta}, r_{\delta}, p_{\delta}\right) \in \Omega \times \mathbf{R} \times \mathbf{R}^{n}$ such that

$$
\lim _{\delta \rightarrow 0} x_{\delta}=x, \lim _{\delta \rightarrow 0}\left(\left|r_{\delta}\right|+\left|p_{\delta}\right|\right)=0,
$$

and

$$
\delta \geqslant \sup _{a \in A\left(x_{\delta}\right)} H_{a}\left(x_{\delta}, u(x)+r_{\delta}, D \phi(x-y) / \varepsilon-\mu \xi+p_{\delta}\right) .
$$

Similarly, we can find $\left(y_{\delta}, s_{\delta}, q_{\delta}\right) \in \Omega \times \mathbf{R} \times \mathbf{R}^{n}$ such that

$$
\lim _{\delta \rightarrow 0} y_{\delta}=y, \lim _{\delta \rightarrow 0}\left(\left|s_{\delta}\right|+\left|q_{\delta}\right|\right)=0,
$$

and

$$
-\delta \leqslant \sup _{a \in A\left(y_{\delta}\right)} H_{a}\left(y_{\delta}, v(y)+s_{\delta}, D \phi(x-y) / \varepsilon-\mu \xi+q_{\delta}\right) .
$$


We first note that it is easy to get a contradiction in the case when $\left(x_{\delta}, y_{6}\right) \in \Omega_{i}$ for $i=1$ or 2 by the same argument as in the proof of Theorem 4.1.

Next, we note that if $x_{\delta} \in \Omega_{1}$ and $y_{\delta} \in \Omega_{2}$ for any $\delta>0$, then we easily obtain a contradiction. Indeed, in this case, from (5.7), we find $a_{\delta} \in A\left(y_{\delta}\right)$ such that

$$
-2 \delta \leqslant H_{a_{\delta}}\left(y_{\delta}, v(y)+s_{\delta}, D \phi(x-y) / \varepsilon-\mu \xi+q_{\delta}\right) \text {. }
$$

For any fixed $r>0$, we find $\widehat{\delta}>0$ such that $A\left(y_{\delta}\right) \subset A_{r}\left(x_{\delta}\right)$ for $\delta \in(0, \widehat{\delta})$. Hence, we can choose $\widehat{a}_{\delta} \in A\left(x_{\delta}\right)$ such that $\left|\widehat{a}_{\delta}-a_{\delta}\right|<r$. Therefore, combining (5.5) with (5.8), by $\left(A 1^{\prime}\right)$, we have

$$
\begin{aligned}
\lambda(u(x)-v(y))-C\left(\left|p_{\delta}\right|+\left|q_{\delta}\right|+\left|r_{\delta}\right|+\left|s_{\delta}\right|\right) & \\
& \leqslant C\left|x_{\delta}-y_{\delta}\right|\left(\frac{|x-y|}{\varepsilon}+\mu\right)+\omega\left(\left|x_{\delta}-y_{\delta}\right|\right)+C r .
\end{aligned}
$$

Sending $\delta \rightarrow 0$ with (5.4) and (5.6) in the above, we have

$$
\lambda(u(x)-v(y)) \leqslant C|x-y|\left(\frac{|x-y|}{\varepsilon}+\mu\right)+\omega(|x-y|)+C r .
$$

Using (5.1) and $\Phi(x, y) \geqslant \gamma$, and sending $\varepsilon \rightarrow 0$ in the above with (5.3), we have $\lambda \gamma \leqslant C r$, which is a contradiction for small $r>0$.

Thus, we may suppose that $x_{\delta} \in \Omega_{2}$ and $y_{\delta} \in \Omega_{1}$ for any $\delta>0$.

Fix $a \in A\left(y_{\delta}\right) \backslash A\left(x_{\delta}\right)$. We claim that there exists $\mu>0$ such that

$$
\lambda v(y)-\left\langle g\left(y_{6}, a\right), D \phi(x-y) / \varepsilon-\mu \xi+q_{\delta}\right\rangle-f\left(y_{6}, a\right)<-\delta
$$

for small $\delta>0$ and $\varepsilon>0$. Indeed, noting $x_{\delta}-y_{\delta} \notin \bigcup_{t>0} B_{t \theta}(t \xi)$ (by (A6)-(1)) and $g\left(y_{\delta}, a\right) \in \bigcup_{t>0} B_{t \theta}(-t \xi)$, we can estimate the left hand side of (5.9) from above by $C+\mu\left\langle g\left(y_{\delta}, a\right), \xi\right\rangle$. Hence, by (A7), we can estimate this from above by

$$
C-\frac{\tau \mu}{2} \sqrt{1-\theta^{2}}
$$

provided $\varepsilon$ and $\delta$ are small enough. Therefore, for a fixed $\mu>0$, we have shown that (5.9) holds.

Noting (5.9) in (5.7), we have

$$
-\delta \leqslant \sup _{a \in A\left(y_{\delta}\right) \cap A\left(x_{\delta}\right)} H_{a}\left(y_{\delta}, v(y)+s_{\delta}, D \phi(x-y) / \varepsilon-\mu \xi+q_{\delta}\right) .
$$

Therefore, combining (5.5) with this, we can proceed by the same argument as in the proof of Theorem 4.1 to get a contradiction. 


\section{REFERENCES}

[1] A.J. Cahill, M.R. James, J.C. Kieffer and D. Williamson, 'Optimal path timing robot manipulators via dynamic programming', (preprint).

[2] M.G. Crandall, H. Ishii and P.-L. Lions, 'User's guide to viscosity solutions of second order partial differential equations', Bull. Amer. Math. Soc. 27 (1992), 1-67.

[3] W.H. Fleming and H. M. Soner, Controlled Markov processes and viscosity solutions (Springer-Verlag, Berlin, Heidelberg, New York, 1992.).

[4] H. Ishii, 'A boundary value problem of the Dirichlet type for Hamilton-Jacobi equations', Ann. Scoula Norm. Sup. Pisa Cl. Sci. 16 (1989), 14-45.

[5] H. Ishii, 'Perron's method for Hamilton-Jacobi equations', Duke Math. J. 55 (1987), 369-384.

[6] H. Ishii and S. Koike, 'A new formulation of state constraint problem of first-order PDEs', SIAM J. Control Optim. (to appear).

[7] S. Koike, 'On the state constraint problem for differential games', Indiana Univ. Math. $J$. (to appear).

Department of Mathematics

Saitama University

Urawa, Saitama 338

Japan

e-mail: koike@ceremade.dauphine.fr 